\title{
Effect of topical loteprednol etabonate with lid hygiene on tear cytokines and meibomian gland dysfunction in prosthetic eye wearers
}

JS Ko ${ }^{1}$, Y Seo ${ }^{1}$, MK Chae ${ }^{1}$, SY Jang ${ }^{2}$ and JS Yoon ${ }^{1}$

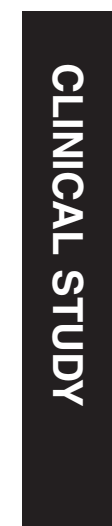

\begin{abstract}
Purpose To assess tear cytokine levels and clinical outcomes in meibomian gland dysfunction (MGD) in the blind eye of patients wearing an ocular prosthesis after 2 months of treatment with topical loteprednol etabonate and eyelid scrubs with warm compresses.

Patients and methods This study included patients with MGD wearing a unilateral ocular prosthesis for more than 1 year. All patients topically received $0.5 \%$ loteprednol etabonate and were instructed to scrub their eyelids with warm compresses on the prosthetic eye for 2 months. We evaluated tear cytokine levels using Multiplex Bead Immunoassays, performed biomicroscopic examination of the lid margins and meibomian gland, conducted meibography imaging, and assessed MGD-related ocular symptoms using a questionnaire for the prosthetic eye before and 2 months after treatment.
\end{abstract}

Results Thirty consecutive patients were included. There were significant reductions in the levels of interleukin (IL)-6, interferon- $\gamma$, monocyte chemotactic protein-1, IL-8, tumor necrosis factor- $\alpha$, and IL-1 $\beta(P<0.001$ for each cytokine). Moreover, there were improvements in ocular symptoms $(P=0.001)$, lid margin abnormalities $(P<0.001)$, meibomian gland expressibility $(P<0.001)$ and meibography findings $(P=0.037)$.

Conclusion Topical loteprednol etabonate in conjunction with eyelid scrubs and warm compresses were effective in treating MGD in prosthetic eye wearers. Furthermore, tear cytokine measurements may serve as an additional approach for evaluating the efficacy of anti-inflammatory treatment for MGD in prosthetic eye wearers.

Eye (2018) 32, 439-445; doi:10.1038/eye.2017.213; published online 20 October 2017

\section{Introduction}

Patients wearing prosthetic eyes often experience symptoms including dryness, irritation, discomfort, and discharge. Indeed, questionnaire-based investigations have reported that most prosthetic eye wearers are concerned about watering, crusting, and discharge in the affected eye. ${ }^{1,2}$ In the clinical setting, many prosthetic eye wearers with ocular discomfort are prescribed artificial lubricants to relieve their ocular symptoms. However, some patients still continue to experience discomfort, even with long-term use of artificial lubricants. ${ }^{3}$

Meibomian gland dysfunction (MGD) is a chronic, diffuse abnormality of the meibomian glands commonly characterized by terminal duct obstruction and/or qualitative and quantitative changes in glandular secretion. ${ }^{4}$ The meibum lipid is modified in patients with MGD, resulting in tear instability, evaporative dry eye, and eyelid inflammation. ${ }^{5,6}$ In our previous study, we demonstrated significant loss of the meibomian gland in eyelids with an ocular prosthesis compared with normal eyes, possibly due to the rubbing of the eyelid margin against the prosthesis, inducing hyperkeratinization and inflammation. ${ }^{3}$

Initial conservative therapy for MGD often includes a combination of warm compresses in addition to eyelid scrubs; however, many patients will not achieve significant improvement with this therapy alone. ${ }^{7}$ Pharmacological therapy for MGD includes
${ }^{1}$ Department of

Ophthalmology, Severance Hospital, Institute of Vision Research, Yonsei University College of Medicine, Seoul, Korea

${ }^{2}$ Department of Ophthalmology, Soonchunhyang Bucheon Hospital, Soonchunhyang University College of Medicine, Bucheon, Korea

Correspondence:

JS Yoon, Department of Ophthalmology, Severance Hospital, Institute of Vision Research, Yonsei University College of Medicine, 50-1 Yonsei-ro, Seodaemun-gu, Seoul 03722, Korea Tel: +82 22228 3570; Fax: +82 23120541 . E-mail: yoonjs@yuhs.ac

Received: 30 November 2016

Accepted in revised form:

21 August 2017

Published online:

20 October 2017 
topical or systemic antibiotics, topical cyclosporine, and topical corticosteroids. ${ }^{7-9}$ Recently, topical loteprednol etabonate was shown to improve symptoms and clinical features by reducing inflammatory cytokine levels in MGD-associated dry eye disease. ${ }^{10-12}$ Topical loteprednol etabonate has the potential side effects of steroid-induced glaucoma and cataract formation, although it is associated with a relatively lower risk of clinically significant increases in intraocular pressure than other conventional corticosteroids. ${ }^{13,14}$ Therefore, clinicians may not use it as a first-line therapy. ${ }^{7}$ However, the aforementioned side effects of steroids are not a concern in prosthetic eye wearers.

The determination of the MGD stage is a fundamental step for planning treatment options and evaluating the efficacy of treatment. ${ }^{12}$ Evaluating the severity of MGD, however, is difficult in the eyelids of the anophthalmic socket compared with the normal eye. The Ocular Surface Disease Index, the most widely used and validated questionnaire, is inapplicable to prosthetic eye wearers because it emphasizes vision-related functions such as reading difficulty and blurred vision. ${ }^{15}$ Moreover, measurements of tear break up time and assessments of ocular surface damage with fluorescent dye staining are impossible in prosthetic eye wearers. On the other hand, tear cytokine levels are correlated with MGD severity because they play an important role in the chronic inflammation associated with MGD. ${ }^{16,17}$ Moreover, the concentration of multiple cytokines significantly decreases after treatment of MGD. ${ }^{12,18}$ Therefore, tear cytokine levels can be used as an objective criterion for diagnosing MGD and analyzing the efficacy of treatment. $^{18}$

In this study, we evaluated improvements in MGD after administration of topical loteprednol etabonate and eyelid scrubs with warm compresses by determining tear cytokine concentrations and multiple clinical parameters. To our knowledge, this is the first study to evaluate the efficacy of topical corticosteroids in the treatment of MGD in prosthetic eye wearers, and the efficacy of MGD treatment in prosthetic eye wearers via measurements of tear cytokine concentrations.

\section{Subjects and methods}

This study included patients diagnosed with obstructive MGD who wore a unilateral ocular prosthesis for more than 1 year. The diagnosis of MGD was based on the presence of ocular symptoms, lid margin abnormalities (irregular lid margin, vascular engorgement, plugged meibomian gland orifices, and anterior or posterior replacement of the mucocutaneous junction), poor meibum expression, and the loss of meibomian glands based on noninvasive meibography. ${ }^{19}$ Because one of the underlying mechanisms of MGD may be the rubbing of the eyelid margin against the prosthesis, ${ }^{3}$ a poorly managed and damaged prosthesis could have a greater impact on the development of MGD. Therefore, this study included only those patients who regularly visited our clinic and kept their prostheses in a fair condition. Patients with inflammation or infection of the socket, or lid abnormalities such as an ectropion or entropion, were excluded from the study. Patients who had socket problems, such as implant exposure or fornix contracture, or a poorly fitted prosthesis were also excluded. Additionally, patients were excluded if they had been using any topical ocular or systemic medication for treatment of MGD or dry eye syndrome, except artificial tears, including topical or oral antibiotics, topical cyclosporine A, topical steroids, topical nonsteroidal anti-inflammatory drugs, and topical ocular allergy medications within the past 3 months.

Thirty patients were enrolled consecutively from the Severance Eye and ENT Hospital, Yonsei University College of Medicine, Seoul, South Korea, between March 2016 and June 2016. All patients topically received 0.5\% loteprednol etabonate (Lotemax; Bausch and Lomb Inc., Rochester, NY, USA), which they applied four times daily following the use of eyelid scrubs with warm compresses twice daily to the prosthetic eye for 2 months. Each patient was asked questions regarding their demographic status and duration of prosthesis use. A chart review was conducted to survey the type of ophthalmic surgery, such as evisceration or enucleation.

This interventional case series was approved prospectively by the Institutional Review Board of Severance Hospital, Yonsei University College of Medicine (Seoul, South Korea), and conducted according to the Declaration of Helsinki and Good Clinical Practices. Informed consent was obtained from all individual participants included in the study.

Ocular symptoms, eyelid margin, meibomian gland expressibility, and meibomian gland loss assessments with meibography were performed in the prosthetic eye and paired normal eye before treatment, and in the prosthetic eye after 2 months of treatment. Tear cytokine analysis was performed in the prosthetic eye before treatment and after 2 months of treatment. Patients were instructed to perform standard eyelid management via face-to-face education and were instructed not to wipe or scrub their eyelid margins on the day of tear sampling. ${ }^{20}$

More than $20 \mu \mathrm{l}$ of tears were obtained without topical anesthesia using a disposable $5-\mu 1$ microcapillary tube from the lower conjunctival sac near the lateral canthus after prosthesis removal. An attempt was made to collect the tear fluid samples without trauma. Tear samples were 
placed in microtubes (Eppendorf, Westbury, NY, USA) and stored at $-70^{\circ} \mathrm{C}$ until further examination. Cytokines were measured using Multiplex Bead Immunoassays (Invitrogen, Carlsbad, CA, USA). The cytokines analyzed were interleukin (IL)-6, interferon- $\gamma$, monocyte chemotactic protein- 1 , IL- 8 , tumor necrosis factor- $\alpha$, and IL-1 $\beta$. The measurements were performed as previously described. ${ }^{21}$ Briefly, $20 \mu \mathrm{l}$ of tear samples were diluted 10 times with assay diluent (Human cytokine 10-plex; Invitrogen). A total volume of $100 \mu 1$ from each sample was used. Cytokine levels were measured with a Luminex 100 TM system (Bio-Rad Laboratories, Hercules, CA, USA). Raw data (mean fluorescent intensity) were analyzed using Bio-Plex Manager Software (Bio-Rad Laboratories) to obtain concentration values.

Ocular symptoms were determined as described previously. ${ }^{3,22}$ To increase the suitability of the questionnaire for measuring ocular symptoms in patients wearing a prosthetic eye, it was modified by omitting visual disturbance factors such as glare, reading difficulty, driving difficulty, and blurred vision.,19 Ocular symptoms were assessed using a five-point categorical scale with the following frequencies: none of the time $(0$ points), some of the time (1), half of the time (2), most of the time (3), and all of the time (4). Patients were asked to assign severity ratings using this scale to the following symptoms: ocular fatigue, discharge, foreign body sensation, dryness, discomfort, sticky sensation, pain, tearing, itching sensation, burning sensation, heavy sensation, excessive blinking, uncomfortable in windy conditions, and uncomfortable in very dry conditions. A history of chalazion or hordeolum was assessed using a three-point categorical scale: no experience ( 0 point), having occurred, but not frequently (2), and frequently occurring (more than once per year) (4). The ocular symptom score was determined by calculating the sum of each individual score, with total possible scores ranging between 0 and 60 points. The score was converted to a score out of 100 .

Microscopic examination of the lid margins and meibomian glands was performed as previously described by one physician who was masked to the clinical information. ${ }^{3}$ The physician scored the presence or absence of the following lid margin abnormalities: irregular lid margin, vascular engorgement, plugged meibomian gland orifices, and anterior or posterior displacement of the mucocutaneous junction. ${ }^{19}$ If any of these signs were present, one point was assigned for each item, with a possible score range of $0-4$ points. Meibomian gland expression was assessed by assigning grades for clarity and ease of meibum expression as follows: clear meibum and easy expression (grade 0), cloudy in response to mild pressure (grade 1), cloudy in response to moderate pressure (grade 2), and no expression in response to hard pressure (grade 3$)^{23}$

LipiView interferometer (TearScience Inc., Morrisville, NC, USA) imaging was performed and meibography scores graded according to meibomian gland loss as described by Arita et al. ${ }^{24}$ The meibography score was graded by one reader who was masked to the clinical information. The meibography score was analyzed in the upper and lower eyelid separately. The total meibography score was calculated as the sum of meibography score of upper eyelid and lower eyelid.

\section{Data analysis}

Statistical analyses were conducted using $\mathrm{R}$ version 3.1.2 (R Foundation, Vienna, Austria). Differences were considered statistically significant when the $P$-values were less than 0.05 . A Wilcoxon signed-rank test was used to compare ordinary categorical variables such as the lid margin abnormality score, meibomian gland expression score, meibography score, and ocular symptom scores. The Wilcoxon signed-rank test was used to compare tear cytokine levels between baseline and after 2 months of treatment because the changes in the concentration of each cytokine before and after treatment were not normally distributed when the Shapiro-Wilk test was performed.

\section{Results}

In total, 30 patients who had used an ocular prosthesis for longer than 1 year were treated with topical loteprednol etabonate four times daily following eyelid scrubs with warm compresses twice daily. The treatment was well tolerated, none of the patients discontinued the study, and no adverse event was noted.

The patients' clinical characteristics are summarized in Table 1. The mean patient age was $46.3 \pm 15.8$ (range, 20-77) years and the mean duration of prosthesis usage was $10.7 \pm 10.2$ (range, $1-40$ ) years. The most common cause of ophthalmic surgery was trauma $(60 \%)$ and evisceration was the most common type of eye removal surgery $(50 \%)$.

The baseline clinical features, meibography findings, and ocular symptom score of the prosthetic eye and paired normal eye are presented in Table 2. The lid margin abnormality score and meibomian gland expression score were significantly higher in the eyelids of prosthetic eyes compared with the normal eyelids $(P<0.001$ for each case; Table 2$)$. Meibography scores were also significantly higher in the eyelids of prosthetic eyes compared with normal eyelids $(P<0.001$; Table 2$)$. 
Table 1 Clinical characteristics

\begin{tabular}{lc}
\hline Variables & Value \\
\hline Total (number of patients) & 30 \\
& \\
Age (years) & $46.3 \pm 15.8$ \\
Mean \pm SD & $20-77$ \\
Range & \\
& \\
Duration of prosthesis use (years) & $10.7 \pm 10.2$ \\
Mean \pm SD & $1-40$ \\
Range & \\
Sex & \\
Women & 16 \\
Men & 14 \\
Cause of blindness: number of patients (\%) & \\
Trauma & $18(60 \%)$ \\
Cancer & $5(17 \%)$ \\
Disease & $5(17 \%)$ \\
Congenital & $2(7 \%)$ \\
Operation: number of patients (\%) & \\
Evisceration & \\
Enucleation & $15(50 \%)$ \\
Unknown & $8(27 \%)$ \\
\hline
\end{tabular}

Abbreviation: SD, standard deviation.

Table 2 Comparison of baseline structural changes in eyelids and ocular symptoms between prosthetic eyes and paired normal eyes

\begin{tabular}{lccc}
\hline & $\begin{array}{c}\text { Normal } \\
\text { eye }\end{array}$ & $\begin{array}{c}\text { Prosthetic } \\
\text { eye }\end{array}$ & P-value \\
\hline $\begin{array}{l}\text { Clinical lid findings } \\
\text { Lid margin abnormality }\end{array}$ & $1(1-2)$ & $2(2-3)$ & $<0.001$ \\
$\begin{array}{l}\text { score } \\
\text { Meibomian gland }\end{array}$ & $1(0-1)$ & $2(1-3)$ & $<0.001$ \\
expression & & & \\
& & & \\
$\begin{array}{l}\text { Meibography findings } \\
\text { Meiboscore, upper eyelid }\end{array}$ & $0(0-1)$ & $1.5(0-3)$ & $<0.001$ \\
$\quad$ Meiboscore, lower eyelid & $0(0-1)$ & $1(1-2)$ & $<0.001$ \\
$\quad$ Meiboscore, total & $1(0-2)$ & $3(2-4)$ & $<0.001$ \\
& 12.5 & 34.2 & 0.001 \\
Ocular symptom score & $(3.4-20.5)$ & $(16.7-51.7)$ & \\
\hline
\end{tabular}

Results are presented as median (interquartile range).

$P$-values were obtained using the Wilcoxon signed-rank test.

Furthermore, the mean ocular symptom score of the eyelids with an ocular prosthesis was significantly higher than that of normal eyelids $(P=0.001$; Table 2$)$.

Table 3 compares the cytokine concentrations of tears from the anophthalmic socket before and after treatment. All tear cytokine concentrations (IL-6, interferon- $\gamma$, monocyte chemotactic protein-1, IL-8, tumor necrosis
Table 3 Analysis of tear cytokine levels before and after treatment of meibomian gland dysfunction in prosthetic eye wearers

\begin{tabular}{lccc}
\hline & Baseline & After 2 months & P-value \\
\hline IL-6 $(\mathrm{pg} / \mathrm{ml})$ & $1451(823-1886)$ & $931.5(513-1463)$ & $<0.001$ \\
IFN- $\gamma(\mathrm{pg} / \mathrm{ml})$ & $611(347-1432)$ & $511(276-1023)$ & $<0.001$ \\
MCP-1 $(\mathrm{pg} / \mathrm{ml})$ & $190.2(154.0-214.9)$ & $143.0(112.0-176.0)$ & $<0.001$ \\
IL-8 $(\mathrm{pg} / \mathrm{ml})$ & $2140(1369-6478)$ & $1638.5(986-5346)$ & $<0.001$ \\
TNF- $\alpha(\mathrm{pg} / \mathrm{ml})$ & $431.5(235-634)$ & $252.5(180-531)$ & $<0.001$ \\
IL-1 $\beta(\mathrm{pg} / \mathrm{ml})$ & $442.5(233-577)$ & $265.0(167-433)$ & $<0.001$ \\
\hline
\end{tabular}

Abbreviations: IFN, interferon; IL, interleukin; $\mathrm{MCP}$, monocyte chemotactic protein; TNF, tumor necrosis factor.

Results are presented as median (interquartile range)

$P$-values were obtained using the Wilcoxon signed-rank test.

factor- $\alpha$, and IL-1 $\beta$ ) significantly decreased after treatment $(P<0.001$ for each cytokine; Table 3$)$. Additionally, clinical lid findings, meiboscores of the lower eyelid, and ocular symptom scores improved significantly after 2 months of treatment with topical loteprednol etabonate and eyelid management $(P<0.001, P=0.037, P=0.001$, respectively; Table 4 ), although meiboscores of the upper eyelid remained unchanged.

\section{Discussion}

The findings of this study suggest that topical loteprednol etabonate with eyelid management for 2 months was effective in improving symptoms and signs of MGD in prosthetic eye wearers. The treatment also significantly decreased the concentration of the inflammatory tear cytokines: IL- 6 , interferon- $\gamma$, monocyte chemotactic protein- 1 , IL- 8 , tumor necrosis factor- $\alpha$, and IL- $1 \beta$. These results were consistent with improved lid margin abnormality, meibomian gland expression, meibography findings, and ocular symptoms.

In our previous study, we demonstrated that the meibomian glands of eyelids with an ocular prosthesis are more likely to be lost, and deterioration of normal anatomical structures might lead to obstructive MGD, which was strongly associated with dry eye ocular symptoms. ${ }^{3}$ Although, the majority of the patients wearing a prosthetic eye report varying degrees of dryness and discomfort, ${ }^{25}$ clinicians and patients have overlooked this discomfort because their primary concern is the health of the remaining eye. ${ }^{1,2}$

Moreover, evaluation of MGD in the anophthalmic socket has multiple limitations compared with the normal eye. First, there is no validated, reliable dry eye questionnaire for prosthetic eye wearers. The Ocular Surface Disease Index, which is the most widely used questionnaire that assesses symptoms in patients with dry eyes, emphasizes vision-related functions such as reading difficulty and blurred vision, which are inapplicable to 
Table 4 Clinical signs and symptoms before and after treatment of meibomian gland dysfunction in prosthetic eye wearers

\begin{tabular}{lccc}
\hline & Baseline & After 2 months & P-value \\
\hline Clinical lid findings & & & \\
$\quad$ Lid margin abnormality score & $2(2-3)$ & $1(1-2)$ & $<0.001$ \\
Meibomian gland expression & $2(1-3)$ & & \\
& & $1.5(0-1)$ & $>0.001$ \\
Meibography findings & $1.5(0-3)$ & $1(1-2)$ & $>0.999$ \\
Meiboscore, upper eyelid & $1(1-2)$ & $3(2-4)$ & 0.037 \\
Meiboscore, lower eyelid & $3(2-4)$ & $23.3(13.3-41.7)$ & 0.037 \\
Meiboscore, total & $34.2(16.7-51.7)$ &
\end{tabular}

Results are presented as median (interquartile range).

$P$-values were obtained using the Wilcoxon signed-rank test.

prosthetic eye wearers. ${ }^{15}$ Measurements of tear break up time and ocular surface staining are also useful tools in the evaluation of the severity of MGD, but also cannot be applied to prosthetic eye wearers. One study reported an improvement in Schirmer test results after treatment of dry eye in prosthetic eye wearers with topical cyclosporine. ${ }^{22}$ Schirmer testing in the anophthalmic socket, however, shows different results compared with the normal eye because the anophthalmic socket lacks the stimulus for reflex tear production due to absence of the cornea. ${ }^{26}$

Noncontact infrared meibography has provided clinicians with objective information of the meibomian gland structure. ${ }^{24}$ Structural loss of the meibomian gland was subjectively scored from grade 0 through to grade 3 , and used as part of the diagnostic criteria for MGD. ${ }^{19}$ The meiboscore, however, may not be a useful tool in evaluating the effect of treatment in MGD because it is difficult to reflect subtle morphologic changes using a grading scale system. Recently, novel image analysis software was introduced for the quantitative and objective measurement of meibomian gland area. ${ }^{27}$ Although several studies reported an increase in the mean ratio of meibomian gland area after treatment, the image analysis requires specific software and complicated image manipulation; therefore, the clinical application may be limited. ${ }^{28,29}$ Moreover, meibography may not be a good tool for evaluating the short-term effects of treatment, because it is unlikely that damaged meibomian gland structures will be restored immediately after treatment.

Currently, there is evidence from multiple studies indicating that MGD induces ocular surface inflammation and this inflammation is associated with the development of MGD. ${ }^{4,17}$ This evidence includes reports of increased tear concentrations of IL- $1 \beta$, IL-6, IL-8, tumor necrosis factor- $\alpha$, and matrix metalloproteinase- 9 in cases of dysfunctional tear syndrome with MGD. ${ }^{30-32}$ Additionally, tear cytokines were shown to be good indicators of clinical improvement after treatment of MGD. ${ }^{12,18,32}$ In this study, we first showed that topical loteprednol etabonate with eyelid management not only controlled eyelid inflammation by decreasing tear cytokine levels but also provided clinical benefits in treating moderate and severe MGD.

The primary limitation of this study is the lack of a control group; therefore, we could not distinguish between the effects of topical loteprednol and lid management. Another limitation is the small number of subjects. The use of nonparametric statistical methods for tear cytokine concentrations can be attributed to the small number of patients. Nevertheless, there was a statistically significant difference in tear cytokine levels and most clinical indicators of MGD after treatment for 2 months. Regarding the duration of prosthetic eye use and the relevance of MGD occurrence, we have previously reported that patients who had worn an ocular prosthesis for more than 10 years had a significantly greater degree of meibomian gland loss compared with those who had not. In this study, both long-term prosthetic eye wearers and short-term wearers responded well to the management regime, so we could not compare the two groups. Further studies regarding stepwise treatment guidelines based on the duration of prosthetic eye use and the degree of MGD will be needed. Despite these limitations, we believe our findings may contribute to further evaluation of the cytokine profiles in prosthetic eye wearers, and promote active treatment of MGD in prosthetic eye wearers.

In conclusion, topical loteprednol etabonate in conjunction with eyelid scrubs and warm compresses was effective in the treatment of MGD in prosthetic eye wearers. Furthermore, tear cytokine measurements can be an additional approach in evaluating the efficacy of antiinflammatory treatment of MGD in prosthetic eye wearers. 


\section{Summary}

What was known before

- Prosthetic eye wearers frequently suffer from meibomian gland dysfunction.

- Evaluating the severity of meibomian gland dysfunction is difficult in the eyelids of the anophthalmic socket.

- Inflammatory tear cytokine levels are correlated with MGD severity.

What this study adds

- Topical loteprednol etabonate in conjunction with eyelid scrubs and warm compresses was effective in the treatment of meibomian gland dysfunction in prosthetic eye wearers.

- Tear cytokine measurements can be an additional approach in evaluating the efficacy of anti-inflammatory treatment of meibomian gland dysfunction in prosthetic eye wearers.

\section{Conflict of interest}

The authors declare no conflict of interest.

\section{Acknowledgements}

This research was supported by the Bio \& Medical Technology Development Program of the NRF funded by the Korean government, MSIP (grant number:

2015M3A9E2067031).

\section{References}

1 Bohman E, Roed Rassmusen ML, Kopp ED. Pain and discomfort in the anophthalmic socket. Curr Opin Ophthalmol 2014; 25: 455-460.

2 Pine K, Sloan B, Stewart J, Jacobs RJ. Concerns of anophthalmic patients wearing artificial eyes. Clin Exp Ophthalmol 2011; 39: 47-52.

3 Jang SY, Lee SY, Yoon JS. Meibomian gland dysfunction in longstanding prosthetic eye wearers. Br J Ophthalmol 2013; 97: 398-402.

4 Nelson JD, Shimazaki J, Benitez-del-Castillo JM, Craig JP, McCulley JP, Den S et al. The international workshop on meibomian gland dysfunction: report of the definition and classification subcommittee. Invest Ophthalmol Vis Sci 2011; 52: 1930-1937.

5 Green-Church KB, Butovich I, Willcox M, Borchman D, Paulsen F, Barabino S et al. The international workshop on meibomian gland dysfunction: report of the subcommittee on tear film lipids and lipid-protein interactions in health and disease. Invest Ophthalmol Vis Sci 2011; 52: 1979-1993.

6 McCulley JP, Shine WE. Meibomian gland function and the tear lipid layer. Ocul Surf 2003; 1: 97-106.

7 Thode AR, Latkany RA. Current and emerging therapeutic strategies for the treatment of meibomian gland dysfunction (MGD). Drugs 2015; 75: 1177-1185.

8 Kashkouli MB, Fazel AJ, Kiavash V, Nojomi M, Ghiasian L. Oral azithromycin versus doxycycline in meibomian gland dysfunction: a randomised double-masked open-label clinical trial. Br J Ophthalmol 2015; 99: 199-204.

9 Akyol-Salman I, Azizi S, Mumcu UY, Ates O, Baykal O. Comparison of the efficacy of topical $\mathrm{N}$-acetyl-cysteine and a topical steroid-antibiotic combination therapy in the treatment of meibomian gland dysfunction. J Ocul Pharmacol Ther 2012; 28: 49-52.

10 Aragona P, Aguennouz M, Rania L, Postorino E, Sommario MS, Roszkowska AM et al. Matrix metalloproteinase 9 and transglutaminase 2 expression at the ocular surface in patients with different forms of dry eye disease. Ophthalmology 2015; 122: 62-71.

11 Kheirkhah A, Dohlman TH, Amparo F, Arnoldner MA, Jamali A, Hamrah P et al. Effects of corneal nerve density on the response to treatment in dry eye disease. Ophthalmology 2015; 122: 662-668.

12 Lee H, Chung B, Kim KS, Seo KY, Choi BJ, Kim TI. Effects of topical loteprednol etabonate on tear cytokines and clinical outcomes in moderate and severe meibomian gland dysfunction: randomized clinical trial. Am J Ophthalmol 2014; 158: 1172-1183. e1171.

13 Holland EJ, Bartlett JD, Paterno MR, Usner DW, Comstock TL. Effects of loteprednol/tobramycin versus dexamethasone/tobramycin on intraocular pressure in healthy volunteers. Cornea 2008; 27: 50-55.

14 Novack GD. Ophthalmic drug development: procedural considerations. J Glaucoma 1998; 7: 202-209.

15 Schiffman RM, Christianson MD, Jacobsen G, Hirsch JD, Reis BL. Reliability and validity of the Ocular Surface Disease Index. Arch Ophthalmol 2000; 118: 615-621.

16 Tong L, Zhou L, Beuerman RW, Zhao SZ, Li XR. Association of tear proteins with Meibomian gland disease and dry eye symptoms. Br J Ophthalmol 2011; 95: 848-852.

17 Suzuki T, Teramukai S, Kinoshita S. Meibomian glands and ocular surface inflammation. Ocul Surf 2015; 13: 133-149.

18 Lee H, Min K, Kim EK, Kim TI. Minocycline controls clinical outcomes and inflammatory cytokines in moderate and severe meibomian gland dysfunction. Am J Ophthalmol 2012; 154: 949-957. e941.

19 Arita R, Itoh K, Maeda S, Maeda K, Furuta A, Fukuoka S et al. Proposed diagnostic criteria for obstructive meibomian gland dysfunction. Ophthalmology 2009; 116: 2058-2063. e2051.

20 Geerling G, Tauber J, Baudouin C, Goto E, Matsumoto Y, O'Brien $\mathrm{T}$ et al. The international workshop on meibomian gland dysfunction: report of the subcommittee on management and treatment of meibomian gland dysfunction. Invest Ophthalmol Vis Sci 2011; 52: 2050-2064.

21 Massingale ML, Li X, Vallabhajosyula M, Chen D, Wei Y, Asbell PA. Analysis of inflammatory cytokines in the tears of dry eye patients. Cornea 2009; 28: 1023-1027.

22 Han JW, Yoon JS, Jang SY. Short-term effects of topical cyclosporine A $0.05 \%$ (Restasis) in long-standing prosthetic eye wearers: a pilot study. Eye (Lond) 2014; 28: 1212-1217.

23 Shimazaki J, Goto E, Ono M, Shimmura S, Tsubota K. Meibomian gland dysfunction in patients with Sjogren syndrome. Ophthalmology 1998; 105: 1485-1488.

24 Arita R, Itoh K, Inoue K, Amano S. Noncontact infrared meibography to document age-related changes of the meibomian glands in a normal population. Ophthalmology 2008; 115: 911-915.

25 Pine KR, Sloan B, Stewart J, Jacobs RJ. The response of the anophthalmic socket to prosthetic eye wear. Clin Exp Optom 2013; 96: 388-393. 
26 Allen L, Kolder HE, Bulgarelli EM, Bulgarelli DM. Artificial eyes and tear measurements. Ophthalmology 1980; 87: 155-157.

27 Arita R, Suehiro J, Haraguchi T, Shirakawa R, Tokoro H, Amano S. Objective image analysis of the meibomian gland area. Br J Ophthalmol 2014; 98: 746-755.

28 Arita R, Suehiro J, Haraguchi T, Maeda S, Maeda K, Tokoro $\mathrm{H}$ et al. Topical diquafosol for patients with obstructive meibomian gland dysfunction. Br J Ophthalmol 2013; 97: 725-729.

29 Arita R, Morishige N, Shirakawa R, Sato Y, Amano S. Effects of eyelid warming devices on tear film parameters in normal subjects and patients with meibomian gland dysfunction. Ocul Surf 2015; 13: 321-330.

30 Acera A, Rocha G, Vecino E, Lema I, Duran JA. Inflammatory markers in the tears of patients with ocular surface disease. Ophthalmic Res 2008; 40: 315-321.

31 Lam H, Bleiden L, de Paiva CS, Farley W, Stern ME, Pflugfelder SC. Tear cytokine profiles in dysfunctional tear syndrome. Am J Ophthalmol 2009; 147: 198-205. e191.

32 Kim JT, Lee SH, Chun YS, Kim JC. Tear cytokines and chemokines in patients with Demodex blepharitis. Cytokine 2011; 53: 94-99. 